\title{
Meta-Analysis of the Pharmacogenetics of ARMS2 A69S Polymorphism and the Response to Advanced Age-Related Macular Degeneration
}

\author{
Jun Zhang ${ }^{a}$ Zhaohui Liu ${ }^{a}$ Shuqiong Hu${ }^{b}$ Jian Qia,c \\ ${ }^{a}$ Chongqing Key Laboratory of Ophthalmology, Chongqing Eye Institute, The First Affiliated Hospital of \\ Chongqing Medical University, Chongqing, PR China; ${ }^{b}$ Department of Ophthalmology, The Jingzhou Aier \\ Eye Hospital, Jingzhou, PR China; 'Department of Ophthalmology, Daping Hospital, Army Medical University, \\ Chongqing, PR China
}

\section{Keywords}

Age-related macular degeneration · ARMS2 - Polymorphism • Anti-vascular endothelial growth factor $\cdot$ Meta-analysis

\begin{abstract}
Age-related macular degeneration (AMD) causes irreversible vision loss, and targeted anti-vascular endothelial growth factor (VEGF) therapy is now the most common and effective treatment. The aim of this meta-analysis is to discuss whether genetic polymorphism of ARMS2 A69S could confer susceptibility to advanced AMD with the response to anti-VEGF treatment. We performed a meta-analysis of relevant published studies selected through electronic databases. A total of 21 preferred studies regarding the association between ARMS2 gene and anti-VEGF treatment response in advanced AMD were generally included in the meta-analysis. The pooled results demonstrated that the carriage of $\mathrm{G}$ allele for ARMS2 A69S presented a better clinical prognosis for advanced AMD treated with anti-VEGF drugs ( $O R=1.38,95 \%$ $\mathrm{Cl}=1.13-1.69, p=0.002$ ). In addition, in the subgroup analysis based on ethnicity, ARMS2 polymorphisms were more likely to be a positive responder for East Asian patients $(\mathrm{OR}=1.67,95 \% \mathrm{Cl}=1.29-2.16, p<0.001)$. This meta-analysis
\end{abstract}

karger@karger.com

(c) 2020 S. Karger AG, Base

www.karger.com/ore

Karger! through a series of rigorous methodology data demonstrated a significant association between ARMS2 A69S polymorphism and the anti-VEGF treatment response in advanced AMD, especially among East Asian population. Numerous well-designed, randomized, multicenter clinical trials with large sample size are required to validate the association.

(c) 2020 S. Karger AG, Basel

\section{Introduction}

Age-related macular degeneration (AMD) is a chronic, progressive condition presenting untreatable in up to $90 \%$ of patients and is a major cause of irreversible blindness in the elderly individuals [1]. AMD primarily affects people aged older than 50 and implicates a population of 30-50 million worldwide [2]. About two-thirds of people aged older than 80 years are afflicted by AMD to some degree in USA [3], while the prevalence of AMD among Asian population aged $40-79$ is estimated at $6.8 \%$ [4]. AMD is classified into 2 types, dry and wet, based on the presence or absence of choroidal neovascularization $(\mathrm{CNV})$ and bleeding, which is further differentiated into exudative AMD (advanced AMD) and geographic atro- 
phy (late "dry" AMD). In advanced AMD, CNV breaks through to the neural retina, causing the leakage of fluid, lipids, and blood, thus leading to fibrous scarring. Although the etiology of AMD is multifactorial, some common risk factors including aging, smoking, gender, hypertension, obesity, and lifetime light exposure have certain epidemiologic associations with its progression [5].

It has been demonstrated that vascular endothelial growth factor (VEGF) could be extracted in choroidal neovascular membranes obtained from AMD patients [6]. Besides, the VEGF levels in the aqueous humor of AMD patients were significantly increased as compared with control eyes without ocular or systemic diseases [7]. In animal models, the expression of VEGF has been identified in experimental $\mathrm{CNV}$, and it presented tendency to induce the neovascularization [8]. Intravitreal treatment with anti-VEGF drugs, such as ranibizumab, aflibercept, and bevacizumab, remains a valid approach to tackle the progression of advanced AMD [9], which dramatically intensifies or stabilizes visual acuity in a large number of patients. Recently, a long time-span prospective study has corroborated that legal blindness attributable to AMD could be reduced by half in some countries when introducing VEGF antagonists [10].

Current targeted therapies for advanced AMD are based on VEGF inhibition. However, we should take into account the potential risk of long-term consequences of intraocular VEGF suppression on the retina due to inhibition of physiological VEGF function. A large community of AMD patients receiving anti-VEGF treatment could not have their lost vision properly improved or recovered, even with persistent macular edema, despite continuous injection of bevacizumab or ranibizumab [11, 12]. Other etiologies such as genetic backgrounds may illustrate the likelihood. Just as Andreoli et al. [13] pointed out that each individual would have a characteristic genome phenotype toward intravitreal injection, we have had an eye on gene polymorphism as to this tough problem.

Though the most significant loci associated with advanced AMD are focused on the gene for complement factor $\mathrm{H}$ located on chromosome 1q31, increasing evidence from the latest large case-control genome-wide association studies confirmed the association with other genes involved as candidate risk factors. The polymorphisms of age-related maculopathy susceptibility 2 (ARMS2, also called LOC387715) gene at the 10q26 locus have been reported to be strongly associated with the inverse effect of hormone replacement therapy on AMD. Investigators have demonstrated that the encoded mRNA and corresponding peptide are expressed in the retina, denoting that ARMS2 transcript takes the responsibility for the relationship with AMD [14]. Besides, the ARMS2 protein has been described as a component of the extracellular matrix and its mRNA owns a unique splice form in the retina but commonly no in other tissues $[15,16]$. Polymorphic site A69S (rs10490924) within the ARMS2 locus was mainly investigated in several studies in regard to AMD, and the results showed strong correlation of poor visual acuity in advanced AMD with the response of anti-VEGF intervention [13].

Although several studies have been reasonably substantiated that ARMS2 A69S polymorphism conferred susceptibility to advanced AMD, others have declared no such associations. We postulated that these disparities are probably triggered by small sample size, ethnic difference, low statistical power, or clinical heterogeneity. In order to better understand the natural association of ARMS2 genetic polymorphism with the anti-VEGF treatment response in advanced AMD, we conducted a systematic meta-analysis. The present study aimed to determine whether ARMS2 A69S polymorphism plays a crucial role in regulating the risk of advanced AMD.

\section{Materials and Methods}

\section{Search Strategy}

A comprehensive online search for relevant studies in the common electronic databases including PubMed, Web of Science, EMBASE, and Cochrane Library was performed by 2 independent researchers for the meta-analysis. Search terms in this study were "gene," "polymorphism," "SNP," "variant," "macular degeneration," "age-related macular degeneration," "AMD," "age-related maculopathy susceptibility 2," "ARMS2," "LOC387715," "antivascular endothelial growth factor," "anti-VEGF," and "anti-angiogenesis." Search syntaxes such as "OR" and "AND" were used between those search terms. The studies were searched without any limitation on language, ethnicity, and publication year. In addition, the reference lists from retrieved studies were also checked to identify the source of any relevant ones that were not yet retrieved by our computerized databases. The final search was upto-date as of November 8, 2019.

\section{Literature Screening}

The available studies retrieved from electronic databases were inspected in terms of the title, keywords, abstract, and full text in our meta-analysis. Any disputes were resolved by group consensus through discussion in collaboration with a third researcher. Studies were conceived eligible for inclusion if they met the following criteria: (a) evaluation on the relationship between ARMS2 A69S polymorphism and the anti-VEGF treatment response in advanced AMD; (b) independent retrospective or prospective study; (c) detailed numbers or percentages in genotype distribution of ARMS2 A69S could be obtained in patients; (d) the study subjects 


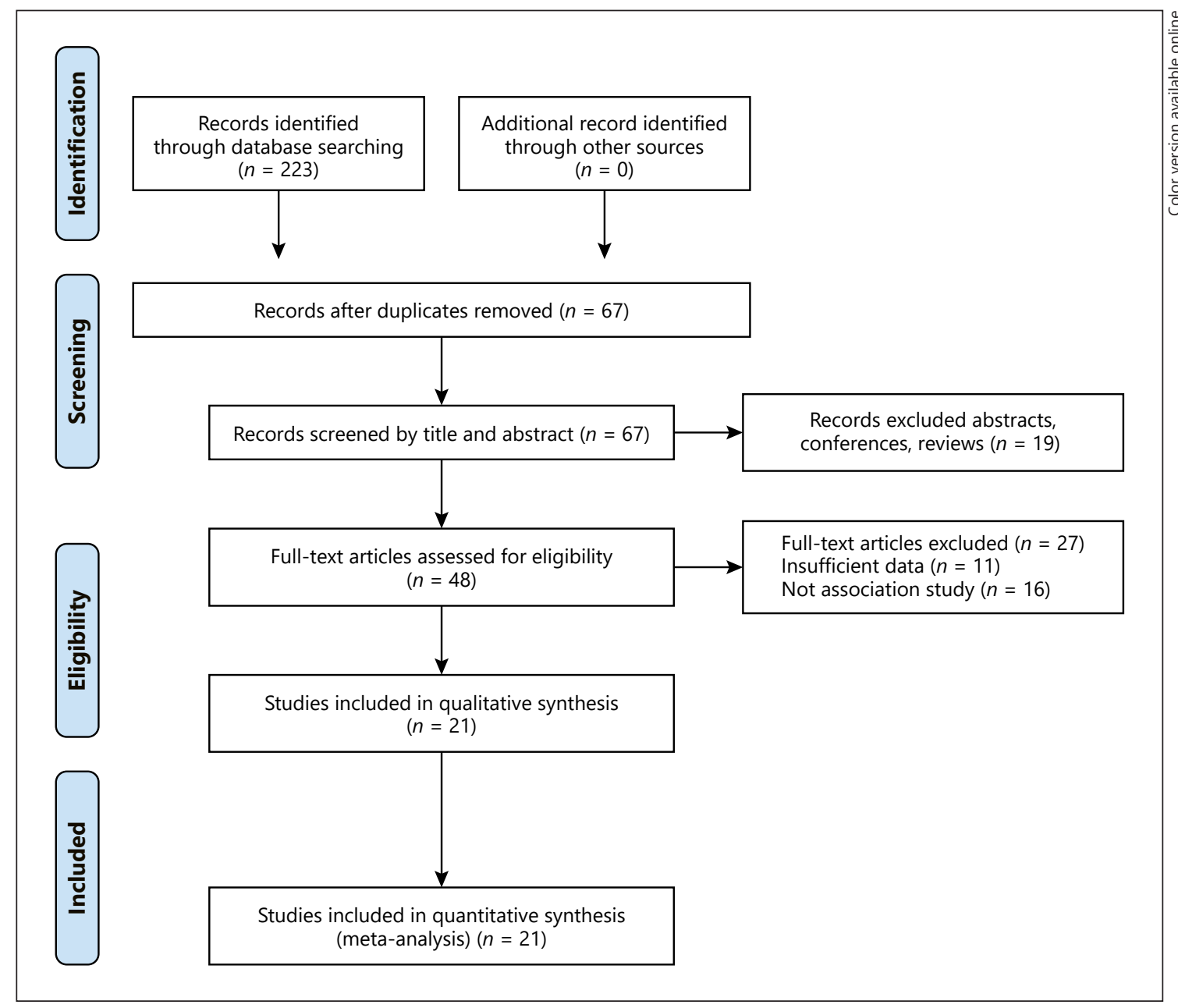

Fig. 1. Flow diagram presenting the result of literature searching process in meta-analysis.

were human beings; and (e) polypoidal choroidal vasculopathy (PCV), as a subtype of advanced AMD, was generally considered in our meta-analysis. Results from previous article, conference, case report, review, opinion, letter, and insufficient raw data after contacting with the corresponding author were extracted and excluded.

\section{Data Extraction and Quality Assessment}

Detailed data were carefully extracted from our eligible studies independently by 2 researchers (J.Z. and Z.L.). The collected data from each study contained first author, publication year, nationality and racial makeup of the study population, study size, characteristics of included cases, information on study design, frequency or percentage in genotype distribution of ARMS2 A69S, treatment modalities, and study endpoint. Patients who underwent the clinical follow-up ranged from 3 to 24 months and at least 3 initial monthly injections of anti-VEGF therapy were required for inclusion. Clinical outcome measures of the positive response to treatment were based on visual acuity and anatomical features of advanced AMD assessed by optical coherence tomography (OCT), fundus fluorescein angiography, and indocyanine green angiogra- phy. The quality evaluation of the selected studies was also performed by 2 researchers (J.Z. and Z.L.) using the Newcastle-Ottawa scale [17]. A study with $>6$ stars was regarded as high quality, and one with 9 stars was regarded as the highest quality. The listed information was rigorously inspected by 2 researchers (J.Z. and Z.L.), and an absolute consensus on all respects of an included paper was reached without any disputes. Any disagreement would be validated and resolved by a third researcher (S.H.).

\section{Statistical Analysis}

A series of quantitative analyses were performed by using the statistical software STATA V.12.0 (StataCorp LP, College Station, TX, USA). The odds ratio (OR) and 95\% confidence interval (CI) were calculated, and pooled ORs were evaluated for the comparison on the frequency of ARMS2 A69S polymorphism in advanced AMD patients. Cochran's $Q$ test was used to identify potential heterogeneity ( $p<0.1$, treated as significant level across all studies). Moreover, the quantitative $I^{2}$ statistic was utilized for evaluation of inconsistency in our meta-analysis, representing the percentage of the observed variability due to heterogeneity rather than chance. The quantification of heterogeneity was assigned of low, moderate, 
Table 1. The general characteristics of all studies included in our meta-analysis

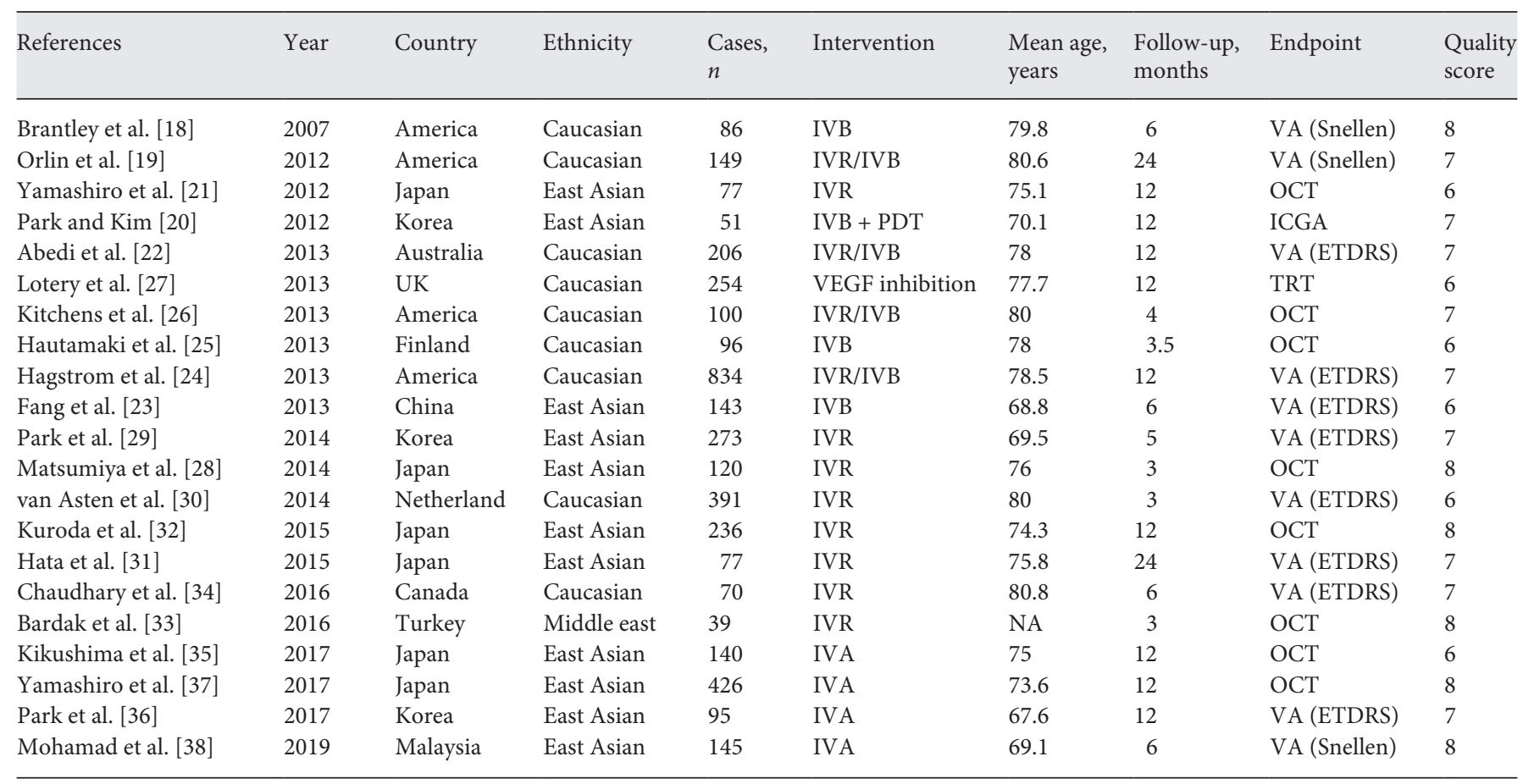

VEGF, vascular endothelial growth factor; OCT, optical coherence tomography; VA, visual acuity; ETDRS, Early Treatment Diabetic Retinopathy Study; IGCA, indocyanine green angiography; TRT, total retinal thickness.

Table 2. The main results of all studies included in our meta-analysis

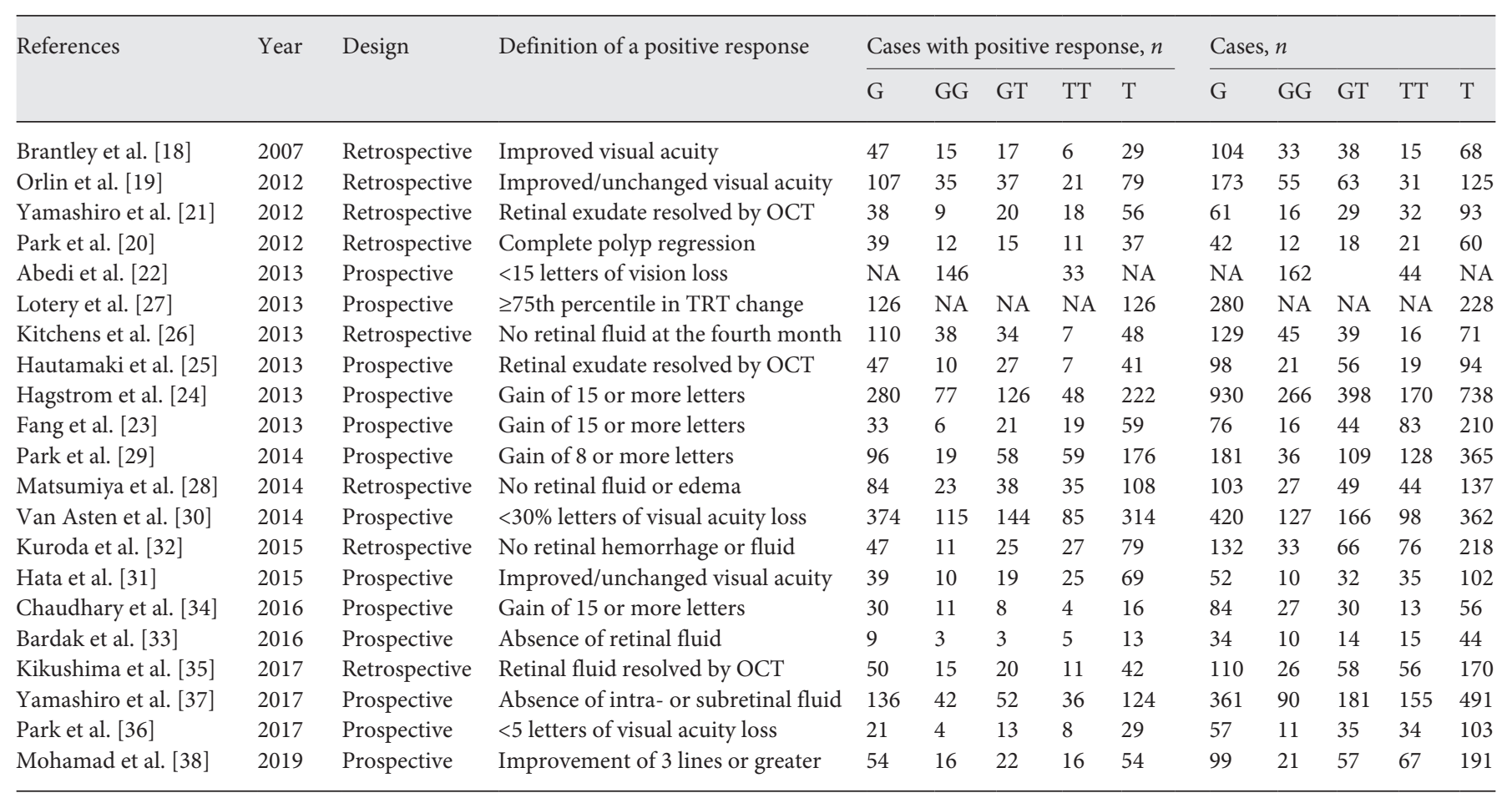

OCT, optical coherence tomography. 
Table 3. The main results of pooled ORs and analysis of ARMS2 gene polymorphism with advanced AMD in our meta-analysis

\begin{tabular}{|c|c|c|c|c|c|c|c|c|}
\hline \multirow[t]{3}{*}{ G versus $\mathrm{T}$ (allelic) } & Overall & 20 & 69.1 & $<0.001$ & $\mathrm{R}$ & 1.38 & $(1.13,1.69)$ & 0.002 \\
\hline & Caucasian & 8 & 55.5 & 0.028 & $\mathrm{R}$ & 1.10 & $(0.86,1.40)$ & 0.455 \\
\hline & East Asian & 11 & 61.1 & 0.004 & $\mathrm{R}$ & 1.67 & $(1.29,2.16)$ & $<0.001$ \\
\hline \multirow{3}{*}{ GG versus TT (homozygote) } & Caucasian & 7 & 31.9 & 0.184 & $\mathrm{~F}$ & 1.25 & $(0.92,1.70)$ & 0.147 \\
\hline & East Asian & 11 & 55.1 & 0.014 & $\mathrm{R}$ & 2.38 & $(1.44,3.93)$ & 0.001 \\
\hline & Middle East & 1 & NA & NA & NA & 0.86 & $(0.15,4.82)$ & 0.861 \\
\hline \multirow[t]{2}{*}{ GT versus TT (heterozygote) } & Overall & 19 & 29.2 & 0.114 & $\mathrm{~F}$ & 1.36 & $(1.14,1.62)$ & 0.001 \\
\hline & Caucasian & 7 & 42.8 & 0.106 & $\mathrm{~F}$ & 1.20 & $(0.91,1.60)$ & 0.201 \\
\hline \multirow{3}{*}{ GG + GT versus TT (dominant) } & Caucasian & 8 & 54.5 & 0.031 & $\mathrm{R}$ & 1.52 & $(0.98,2.35)$ & 0.063 \\
\hline & East Asian & 11 & 40.6 & 0.078 & $\mathrm{R}$ & 1.76 & $(1.31,2.36)$ & $<0.001$ \\
\hline & Middle East & 1 & NA & NA & NA & 0.67 & $(0.16,2.75)$ & 0.575 \\
\hline \multirow[t]{4}{*}{ GG versus GT + TT (recessive) } & Overall & 19 & 47.3 & 0.012 & $\mathrm{R}$ & 1.51 & $(1.15,2.00)$ & 0.004 \\
\hline & Caucasian & 7 & 0.0 & 0.718 & $\mathrm{~F}$ & 1.09 & $(0.87,1.38)$ & 0.460 \\
\hline & East Asian & 11 & 53.3 & 0.018 & $\mathrm{R}$ & 1.90 & $(1.21,2.98)$ & 0.005 \\
\hline & Middle East & 1 & NA & NA & NA & 1.13 & $(0.23,5.45)$ & 0.884 \\
\hline
\end{tabular}

AMD, age-related macular degeneration; OR, odds ratio; CI, confidence interval; NA, not available.

high, and extremely high to $I^{2}$ values of $0-25,25-50,50-75$, and $75-100 \%$, respectively. Either the fixed-effect model $\left(I^{2}<50 \%\right.$ and $p>0.1)$ or random-effect model $\left(I^{2} \geq 50 \%\right.$ and $\left.p<0.1\right)$ was applied for the pooled ORs and 95\% CIs according to the heterogeneity. We also performed sensitivity analysis to assess the effect of each study on the pooled ORs by omitting one study in each turn. Moreover, subgroup analysis was executed to apprehend the strength of association between different ethnicities. A series of meta-regression analyses were applied for the discussion of main heterogeneity. Publication bias was estimated by Begg's test with the funnel plot and Egger's regression test. $p$ value $<0.05$ was considered statistically significant in these comparisons.

\section{Results}

\section{General Characteristics of Selected Studies and Quality Assessment}

A flow diagram of the study selection process is presented in Figure 1. The search strategy resulted in 223 relevant articles from the 4 databases. Of which, 175 studies were excluded for duplication (156 articles) and ineligible categories such as abstract, review, conference, and case report (19 articles). The rest 48 full-text articles were left in our meta-analysis. Among them, 11 studies did not provide detailed genotype results after perusing the documents and additional files and 16 articles did not discuss the association between ARMS2 and anti-VEGF intervention. Finally, a total of 21 preferred studies regarding the association between ARMS2 gene and the anti-VEGF treatment response in advanced AMD were generally included in the current meta-analysis [18-38]. The main characteristics and data of the enrolled studies are listed in Table 1. Regarding ethnicity, 9 of these studies were performed in Caucasian, 11 in East Asian, and 1 in Middle East people. Four studies used aflibercept, 3 used bevacizumab, and 8 used ranibizumab, while 4 studies used either ranibizumab or bevacizumab, one used bevacizumab or photodynamic therapy, and another one used VEGF inhibition. The follow-up duration ranged from 3 months to 2 years. We classified the definition of positive or negative response based on the improvement on visual acuity or macular morphological changes. Moreover, the quality assessment of all included studies is presented in the Table 1 and all studies were of high quality with $>6$ stars. 


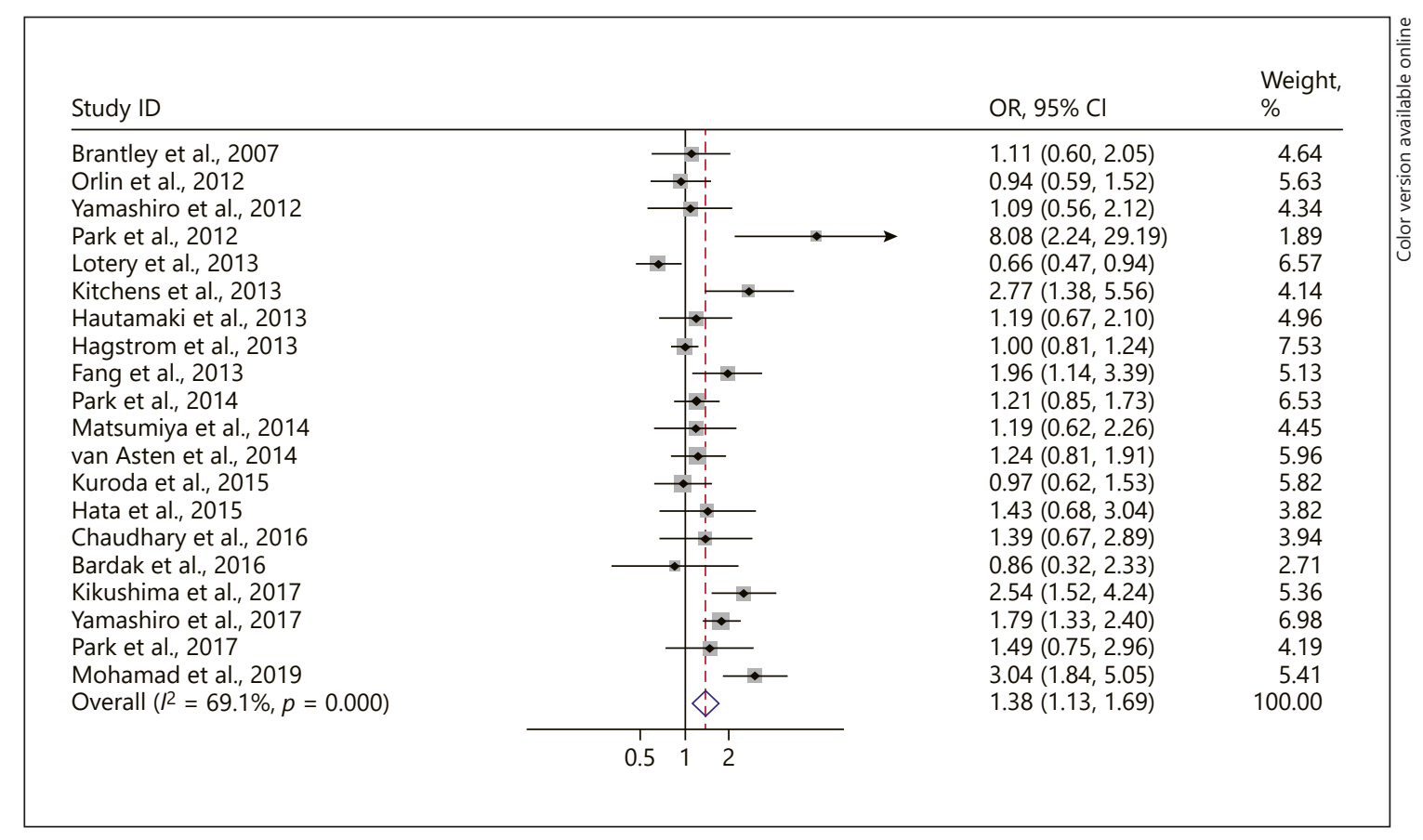

Fig. 2. Evaluation of the association between ARMS2 gene polymorphism (G vs. T) with advanced AMD. AMD, age-related macular degeneration.

\section{Bias Assessment of the Included Studies}

The evaluation of potential bias was primarily expounded in Table 2. Thirteen studies were prospective, and 8 were retrospective. Overall, the quality of the included studies was consistently robust. Of the studies, there were no obvious biases in the selection of advanced AMD patients, confounding, or selective outcome reports. The result with definition of a positive responder could be validated in our meta-analysis.

\section{Association between ARMS2 A69S Polymorphism} and Advanced AMD Susceptibility with Anti-VEGF

\section{Treatment}

We meta-analyzed the 21 included studies for the pooled associations between treatment response in advanced AMD and ARMS2 A69S genotypes in Table 3. We summarized the statistical differences involving 5 genotype comparisons (19 studies for G vs. T, GG vs. TT, and GT vs. TT; 20 studies for GG + GT vs. TT and GG vs. GT $+\mathrm{TT}$ ) of the study population (allelic model: $\mathrm{OR}=1.38$, $95 \% \mathrm{CI}=1.13-1.69, p=0.002$, random model; homozygote model: $\mathrm{OR}=1.88,95 \% \mathrm{CI}=1.32-2.67, p<0.001$, random model; heterozygote model: $\mathrm{OR}=1.36,95 \% \mathrm{CI}$ $=1.14-1.62, p=0.001$, fixed model; dominant model: OR $=1.62,95 \% \mathrm{CI}=1.27-2.06, p<0.001$, random model; re- cessive model: $\mathrm{OR}=1.51,95 \% \mathrm{CI}=1.15-2.00, p=0.004$, random model) (Fig. 2-6). Besides, subgroup analysis based on 3 different racial groups revealed that there were no significant differences between any Caucasian or Middle East genotypic comparison models, but a significant association was indicated in the pooled analyses between A69S and anti-VEGF effects in all genotypic comparisons for East Asian participants (allelic model: $\mathrm{OR}=1.67,95 \%$ $\mathrm{CI}=1.29-2.16, p<0.001$, random model; homozygote model: $\mathrm{OR}=2.38,95 \% \mathrm{CI}=1.44-3.93, p=0.001$, random model; heterozygote model: $\mathrm{OR}=1.50,95 \% \mathrm{CI}=1.19$ $1.62, p=0.001$, fixed model; dominant model: $\mathrm{OR}=1.62$, $95 \% \mathrm{CI}=1.27-2.06, p<0.001$, random model; recessive model: $\mathrm{OR}=1.51,95 \% \mathrm{CI}=1.15-2.00, p=0.004$, random model) (Table 3).

\section{Heterogeneity Test and Sensitivity Analysis}

Significant heterogeneity between the A69S polymorphism in the ARMS2 gene and the anti-VEGF treatment response in advanced AMD was observed among 4 genotype comparisons (all $p<0.1$ ) (Fig. 2, 3, 5, 6). Our subgroup analysis in the homozygote and the recessive model suggested the primary heterogeneity owed to racial origin (Table 3). The pooled ORs for sensitivity analysis remained substantial when omitting 1 study at each time. 


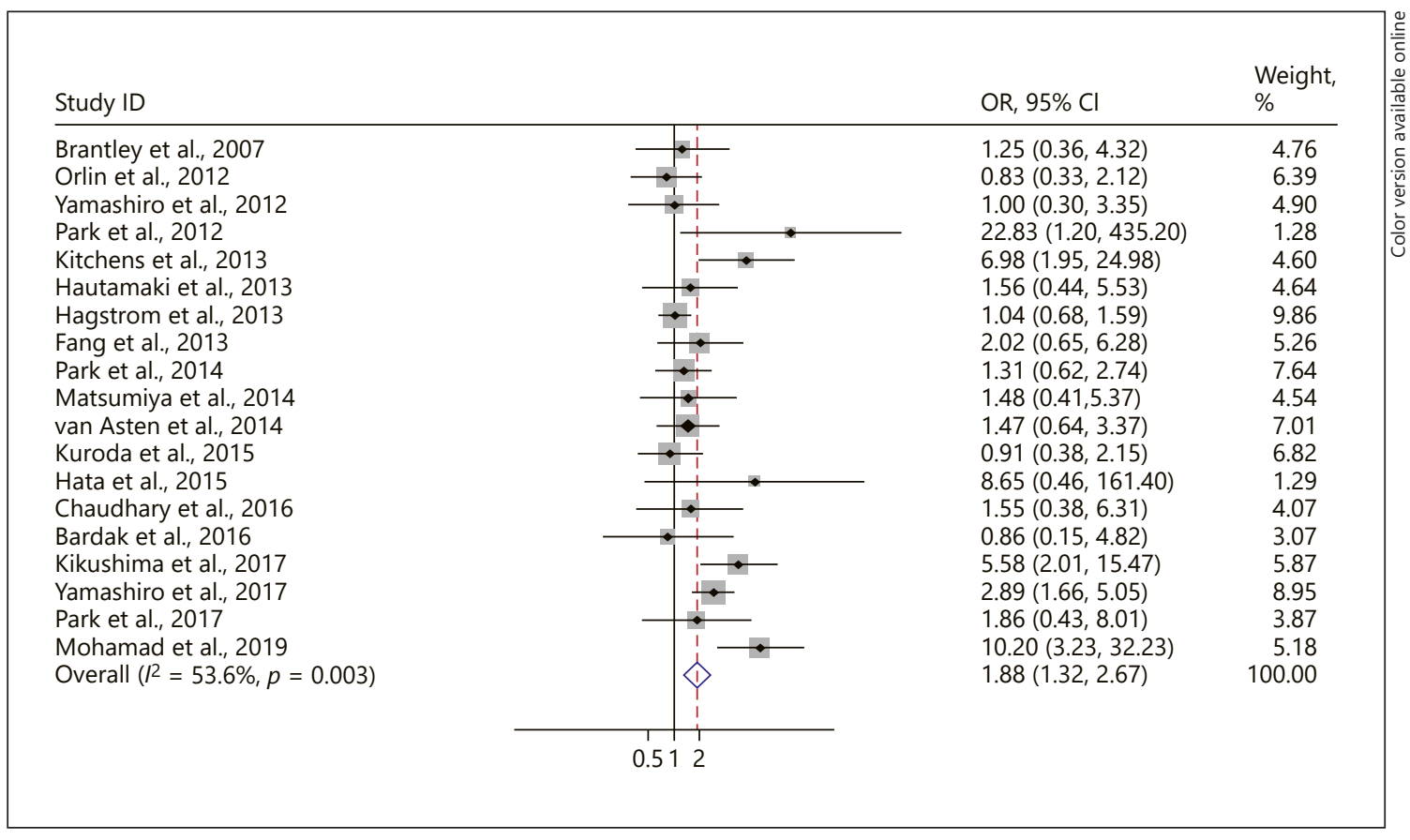

Fig. 3. Assessment of the association between ARMS2 gene polymorphism (GG vs. TT) with advanced AMD. AMD, age-related macular degeneration.

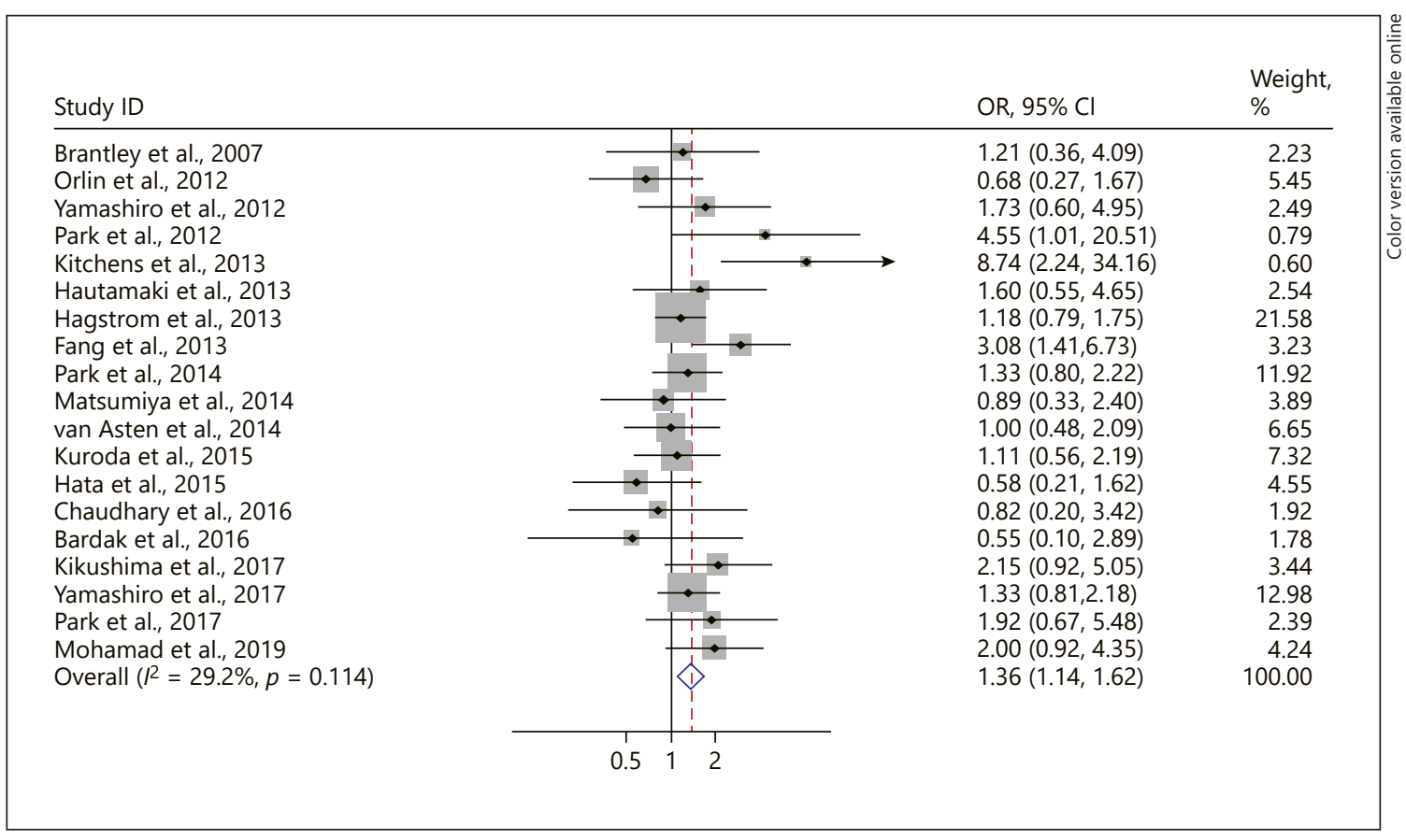

Fig. 4. Estimation of the association between ARMS2 gene polymorphism (GT vs. TT) with advanced AMD. AMD, age-related macular degeneration. 


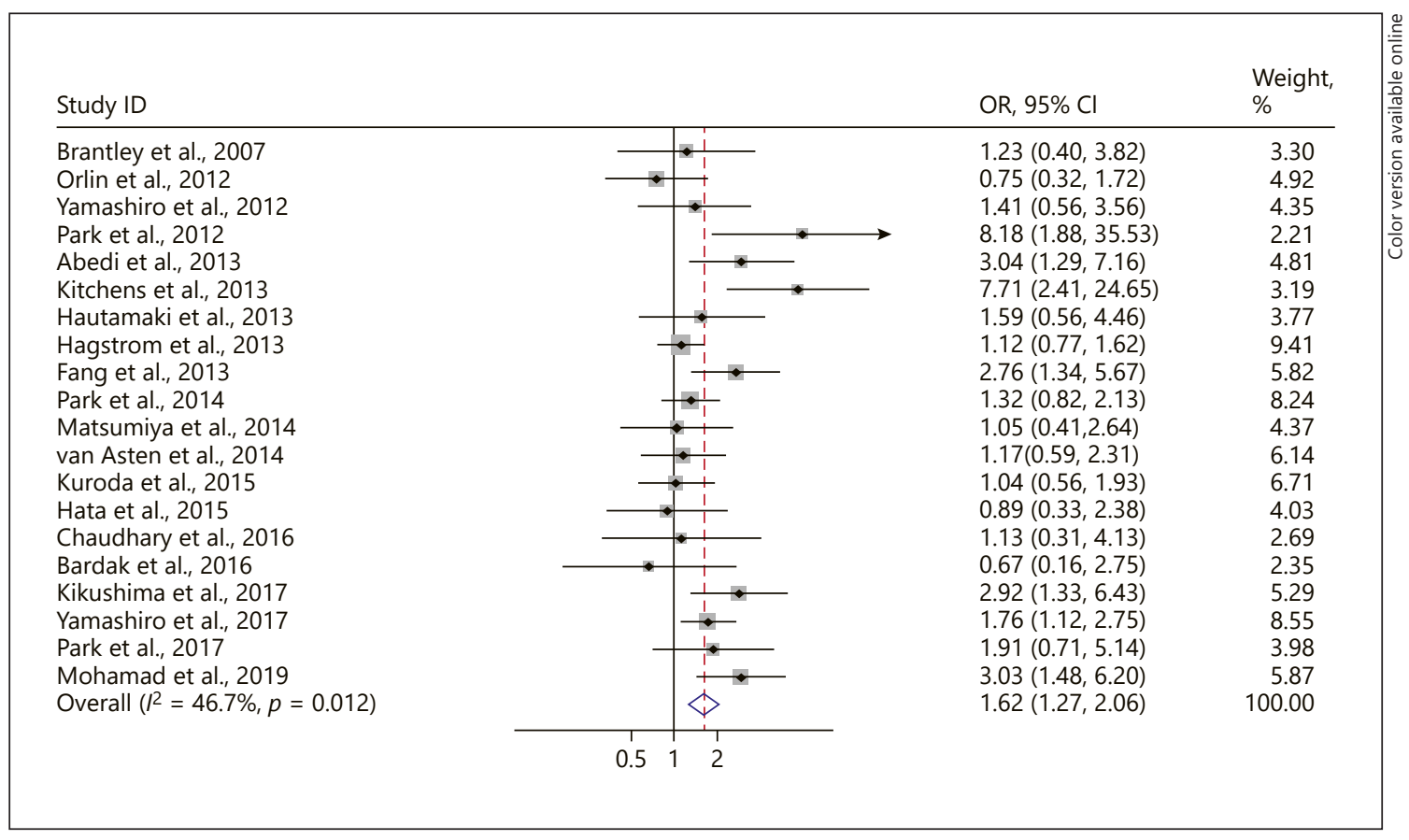

Fig. 5. Evaluation of the association between ARMS2 gene polymorphism (GG + GT vs. TT) with advanced AMD. AMD, age-related macular degeneration.

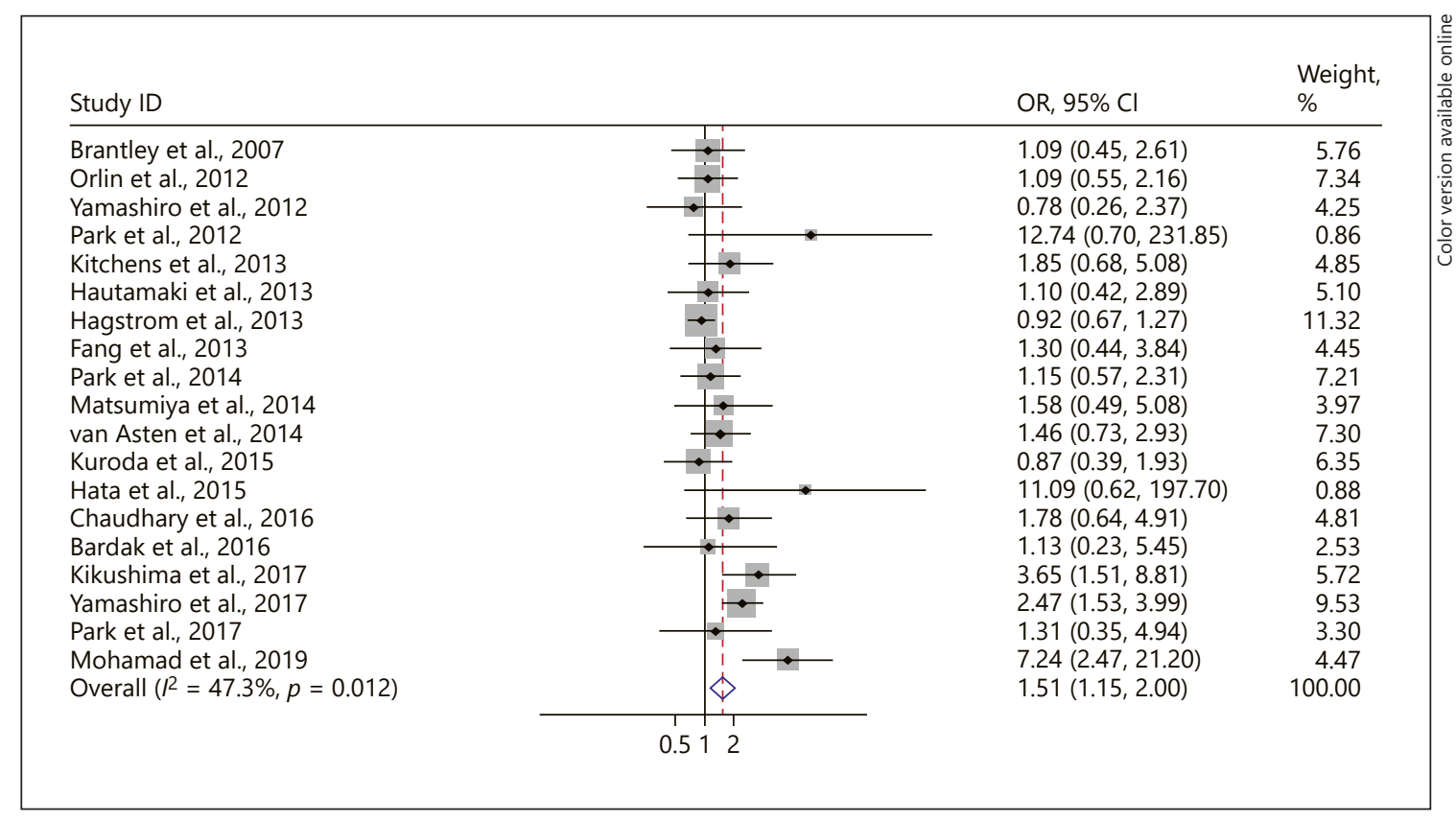

Fig. 6. Assessment of the association between ARMS2 gene polymorphism (GG vs. GT + TT) with advanced AMD. AMD, age-related macular degeneration. 
Fig. 7. Assessment of the sensitivity analysis between ARMS2 gene polymorphism ( $\mathrm{G}$ vs. T) with advanced AMD. AMD, agerelated macular degeneration.
Meta-analysis random-effects estimates (exponential form) Study ommited

Brantley et al., 2007

Orlin et al., 2012

Yamashiro et al., 2012

Park et al., 2012

Lotery et al., 2013

Kitchens et al., 2013

Hautamaki et al., 2013

Hagstrom et al., 2013

Fang et al., 2013

Park et al., 2014

Matsumiya et al., 2014

van Asten et al., 2014

Kuroda et al., 2015

Hata et al., 2015

Chaudhary et al., 2016

Bardak et al., 2016

Kikushima et al., 2017

Yamashiro et al., 2017

Park et al., 2017

Mohamad et al., 2019

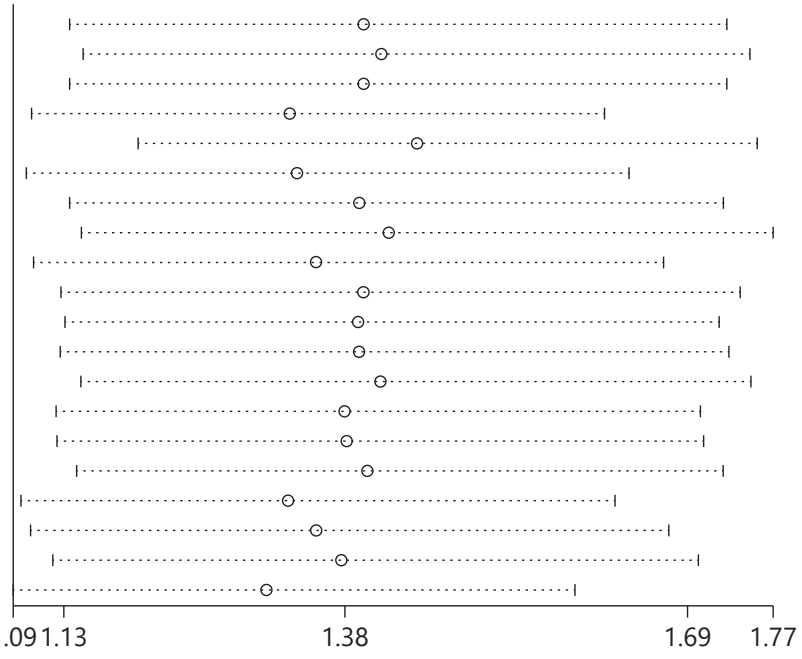

Table 4. Meta-regression for the heterogeneity of $\mathrm{G}$ versus $\mathrm{T}$ comparison in our meta-analysis

\begin{tabular}{lllll}
\hline Covariate factors & $\operatorname{Exp}(\mathrm{b})$ & Std. Err & $p$ value & 95\% CI \\
\hline Design & 1.33 & 0.25 & 0.052 & $0.99-1.78$ \\
Ethnicity & 2.52 & 0.75 & 0.006 & $1.35-4.71$ \\
Follow-up, months & 1.74 & 0.60 & 0.130 & $0.84-3.60$ \\
Intervention & 1.02 & 0.33 & 0.944 & $0.52-2.01$ \\
Mean age, years & 4.14 & 5.75 & 0.319 & $0.22-76.4$ \\
Cases, $n$ & 2.34 & 0.71 & 0.011 & $1.24-4.41$ \\
\hline
\end{tabular}

Our results demonstrated that no study absolutely changed the relationship between ARMS2 A69S and treatment response in advanced AMD, signifying that the pooled ORs of the present meta-analysis were relatively robust (Fig. 7, others in online suppl. Fig. 1-4; see www. karger.com/doi/10.1159/000508738 for all online suppl. material).

\section{Meta-Regression}

We tried to apply meta-regression for analyzing the heterogeneity of $\mathrm{G}$ versus $\mathrm{T}$ genotype to the greatest extent. The study design, ethnicity, follow-up duration, intervention, mean age, and study size were thoroughly treated as independent covariate factors. As delineated in Table 4, our meta-regression analysis showed no significant correlations between the aforementioned variables except ethnicity and number of cases (for ethnicity: $p=$ $0.006,95 \% \mathrm{CI}=1.35-4.71$; for number of cases: $p=0.011$, $95 \% \mathrm{CI}=1.24-4.41)$.

\section{Publication Bias}

Begg's test with the funnel plot and Egger's regression test were performed to assess the source of publication bias between ARMS2 A69S and the treatment response in advanced AMD. There were no conspicuous asymmetries of funnel plots in any genotypic comparisons (Fig. 8, others in online suppl. Fig. 5-8); moreover, no statistically significant relationship was found in Egger's regression test, suggesting no apparent evidence of publication bias $(p>0.05$, Table 5$)$.

\section{Discussion}

AMD is the predominant cause for irreversible blindness in senile population worldwide, especially the advanced type. Anti-VEGF treatment is the primary therapy for patients with vision loss caused by advanced AMD $[39,40]$. A total of 13 prospective and 8 retrospective studies involving 4,008 cases with the association between anti-VEGF and A69S were included in our current meta-analysis. Our results revealed that the ARMS2 A69S allele might be a better prognostic factor for advanced AMD. Specifically, the pooled results based on ethnicity 


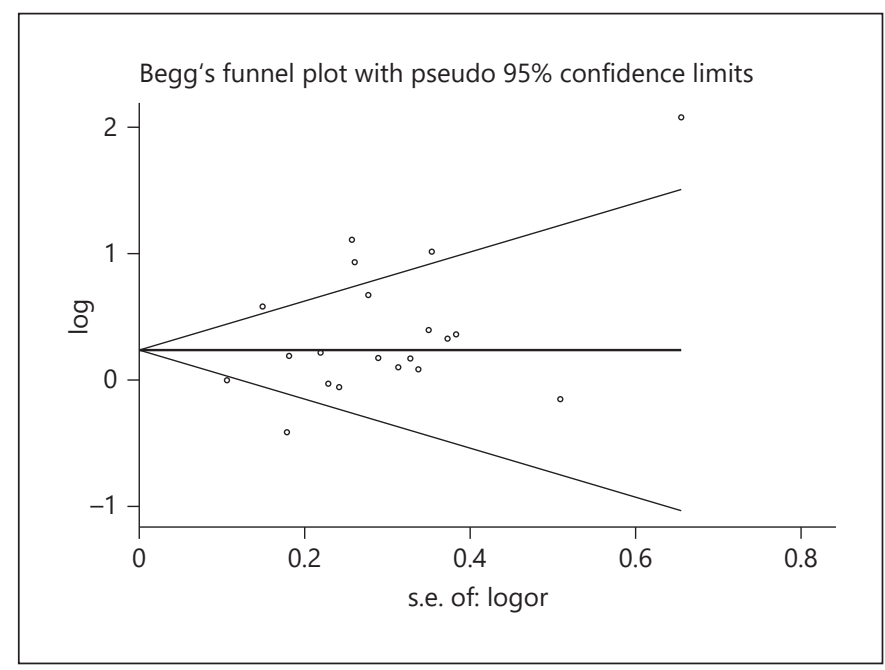

Fig. 8. Estimation of the publication bias between ARMS2 gene polymorphism (G vs. T) with advanced AMD. AMD, age-related macular degeneration.

indicated that there was a significant association between ARMS2 A69S and anti-VEGF treatment response in exudative AMD in the East Asian group, but not in the Caucasian or Middle East group. Thus, these findings may enable clinicians to make the best of these effective medications, at least for East Asian patients.

Anti-VEGF treatment has been extended to treat eyes with PCV, which is a more common subtype of exudative AMD in Asians compared with the Caucasians. In our meta-analysis, a group of patients investigated by Park and Kim [20] had considered PCV subtype and received combined treatment consisting of photodynamic therapy with intravitreal bevacizumab and another group of patients in the same condition explored by Hata et al. [31] was treated with ranibizumab. Besides, Park et al. [36] only centered pharmacogenetics on the association of ARMS2 A69S polymorphism with ranibizumab treatment in PCV. The lack of thoroughly exudative AMD clinical subtypes is one of the major limitations as performing analysis based on the CNV subtypes (PCV and neovascular AMD) could provide more specific information on which exudative AMD is related with the different response to anti-VEGF therapy. The remaining antiVEGF pharmacogenetic studies of exudative AMD did not precisely delineate whether PCV was considered in the total of collected genotypic data. Unfortunately, we could not rule out the accurate proportion of $\mathrm{PCV}$ in the overall exudative AMD patients, though considering that A69S showed significant association only in the East
Table 5. Bias between ARMS2 genetic polymorphism with advanced AMD in our meta-analysis

\begin{tabular}{llll}
\hline $\begin{array}{l}\text { Polymorphism } \\
\text { (rs10490924) }\end{array}$ & $\begin{array}{l}\text { Publication, } \\
n\end{array}$ & \multicolumn{2}{l}{ Publication bias $(p$ value) } \\
\cline { 3 - 4 } & & Begg's test & Egger's test \\
\hline G versus T & 20 & 0.206 & 0.092 \\
GG versus TT & 19 & 0.142 & 0.125 \\
GT versus TT & 19 & 0.889 & 0.455 \\
GG + GT versus TT & 20 & 0.456 & 0.240 \\
GG versus GT + TT & 19 & 0.142 & 0.075
\end{tabular}

AMD, age-related macular degeneration.

Asian population in this meta-analysis. Furthermore, our results may be partly explained by the use of different anti-VEGF drugs during treatment, inconsistent follow-up endpoint, discrepant dosing intervals, and adjusting for different environmental factors across studies. However, being consistent with the positive summarized effect, removing the genotype of the cases with PCV did not exert any influence on the overall comparison (allelic model: $\mathrm{OR}=1.33,95 \% \mathrm{CI}=1.08-1.63$; homozygote model: $\mathrm{OR}=1.77,95 \% \mathrm{CI}=1.24-2.55$; heterozygote model: $\mathrm{OR}=1.36,95 \% \mathrm{CI}=1.13-1.63$; dominant model: $\mathrm{OR}=$ $1.58,95 \% \mathrm{CI}=1.24-2.02$; recessive model: $\mathrm{OR}=1.46$, $95 \% \mathrm{CI}=1.10-1.94)$ due to its small sample size $(n=51$, $n=77$, and $n=95$, respectively).

Obviously, considerable genetic heterogeneity existed in our current study, though the results of sensitivity analysis by removing 1 study in each turn showed that the present meta-analysis was relatively robust. Our subgroup analysis based on involved races in the study population suggested that heterogeneity is particularly pronounced among East Asian and Middle East population. Meta-regression was carried out to explore the heterogeneity of $G$ versus $T$ allele, and the results indicated that ethnicity and number of cases were the origin of heterogeneous factors. In addition, heterogeneity may also have arisen from the genetic background, sex difference, lifestyle, level of socioeconomic development, genotyping method, and definition of response.

It has been verified that ARMS2 lies in the AMD susceptibility locus identified on chromosome 10q26 and is commonly expressed in the retina. Evidence has suggested that ARMS2 variants could bring about dysfunction of the retinal pigment epithelium due to mitochondrial DNA damage that accumulates in the retina and its pigment epithelium. Sobrin et al. [41] suggested that genetic 
mutation at this locus has conferred a differential risk for $\mathrm{CNV}$ versus geographic atrophy. With respect to the possible biological mechanism, the ARMS2 gene product has been localized to the mitochondrial outer membrane; it has been proposed that the A69S polymorphism could alter ARMS2 function and increase the susceptibility of photoreceptor cells to oxidative damage and aging [14]. In addition, the cellular mitochondria are known to be a major source of superoxide anion, implying that damage to the mitochondria would bring about oxidative stress in neovascular AMD possibly affecting the response to antiVEGF inhibitors. Besides, Kortvely et al. [15] reported that the ARMS2 protein is mostly confined to choroid pillars in human eyes, corresponding to the principal sites of drusen deposition. Accordingly, abnormal expression of ARMS2 would exhibit higher susceptibility to the development of drusen and AMD. As such, it is understandable to increase the risk of developing AMD, but the role of nonsynonymous A69S single-nucleotide polymorphism in the pathogenesis of influencing response to anti-VEGF treatment remains unknown. Abedi et al. [22] implied that the loss of structural integrity of Bruch's membrane, driven by oxidative pathways mediated by ARMS2 A69S variants, may largely contribute to the underlying biological mechanisms for a weak response to intravitreal anti-VEGF treatment.

Yamashiro et al. [37] argued that ARMS2 A69S was not significantly associated with the changes in visual acuity or anatomic outcomes during the 1-year anti-VEGF treatment in treatment-naïve advanced AMD patients, while Kitchens et al. [26] confirmed that A69S was positively correlated with the achievement of dry maculae after 3 monthly injections of ranibizumab or bevacizumab, suggesting better response of ARMS2 A69S with advanced AMD. Moreover, Teper et al. [42] indicated a significant influence of the risk homozygosis (TT) for ARMS2 A69S on the visual response after ranibizumab treatment. The differences could be owed to the small sample size and relatively short-term follow-up period in those studies. We have a large enough sample size containing 4,008 cases in the current meta-analysis; the results strongly demonstrated that $G$ allele carriage or GG homozygosis might be useful for better predicting visual outcomes after anti-VEGF treatment.

Apart from ARMS2 A69S polymorphism, several variants within the chromosome 10q26 region have been investigated and their association with advanced AMD susceptibility has been demonstrated, such as a HTRA1 promoter variant rs 11200638 adjacent to $A R M S 2$ and an insertion/deletion variant in the $3^{\prime}$ untranslated region of
ARMS2 gene [43]. Moreover, the functional rs11200638 in the promoter region of the HTRA1 gene exhibited a complete linkage disequilibrium $\left(\mathrm{D}^{\prime}>0.99\right)$ with $A R M S 2$ A69S [44]. However, and by contrast with our results, evidence from a meta-analysis by Zhou et al. [45] showed no influence of pharmacogenetic response with HTRA1 rs 11200638 . Some of this inconsistency may be accounted for by the following reasons that the number of eligible studies and included patients by Zhou et al. [45] was less than that in the current study ( 5 studies vs. 21 studies; 1,570 cases vs. 4,008 cases). In addition, other factors such as epigenetic or environmental conditions also appeared to play a role in the discrepancy. More studies and further rigorous investigations are needed to address this issue.

A previous meta-analysis conducted by $\mathrm{Hu}$ et al. [46] has revealed a significant association between ARMS2 A69S and the response to anti-angiogenesis treatment in advanced AMD patients among 3 genotypic models (homozygote model: $\mathrm{OR}=1.34,95 \% \mathrm{CI}=1.01-1.77, p=$ 0.039; heterozygote model: $\mathrm{OR}=1.58,95 \% \mathrm{CI}=1.08-$ 2.31, $p=0.018$; dominant model: $\mathrm{OR}=1.74,95 \% \mathrm{CI}=$ $1.19-2.52, p=0.004)$. Consistently, our meta-analysis also found the significance of the difference in the comparison of $\mathrm{G}$ versus $\mathrm{T}(\mathrm{OR}=1.38,95 \% \mathrm{CI}=1.13-1.69$, $p=0.002$ ). Moreover, our meta-analysis focused on 3 ethnicities including East Asian, Caucasian, and Middle East population, though no significant association was validated in last 2 races. Furthermore, the pooled results in the current study did not delineate any inconsistency in the exploration of sensitivity test and publication bias, which differs from the conclusions from $\mathrm{Hu}$ et al. [46]. The explanation for this difference could be drawn on the increasing number of studies and larger sample size, thus guaranteeing our cumulative ORs higher definiteness, stability, and accuracy than before.

In order to accurately interpret our combined results, several potential limitations of the current study should not be ignored. First, heterogeneity based on meta-regression was found in covariate factors including ethnicity and number of cases. Second, the variable criteria in grouping the positive and negative response to the antiVEGF treatment could also be one of the factors affecting the pharmacogenetic results, as there are contradictory findings in previous studies, though the included ones were given adequate definition of positive and negative responses. Third, the number of patients was relatively small in each study; thus, a research with larger sample size involving different ethnic regions is necessary for further analysis. Fourth, the difference between central 
macular thickness and maximum lesion thickness with baseline level was also a meaningful clinical outcome in responsive to anti-VEGF treatment. Unfortunately, our study could not point out any relationship between these 2 clinical outcomes and the polymorphisms. Fifth limitation is that only 3 ethnic backgrounds with relatively small studies were included in this meta-analysis; thus, further efforts to lower the incidence of ethnic bias will be taken into account. Finally, included studies were of high quality, but presenting a challenge that the nonrandomized study design was not considered in this metaanalysis.

\section{Conclusion}

The current meta-analysis demonstrated a series of rigorous methodology data for a significant association between ARMS2 A69S and the anti-VEGF treatment response in advanced AMD, especially in East Asian population. More studies, well-designed work with randomized, multicenter clinical trials or intervention studies incorporating different ethnicities together with gene-gene or gene-environment interaction, are needed to further prove the biofunctional role of this common ARMS2 polymorphism.

\section{Statement of Ethics}

All analyses were based on previous published studies; thus, no ethical approval and patient consent are required.

\section{Conflict of Interest Statement}

The authors declare that they have no conflicts of interest to this work.

\section{Funding Sources}

This work was supported by the National Natural Science Foundation Project (Grant No. 81770916 and 81400389) and Chongqing Science \& Technology Foundation Project (Grant No. cstc2014jcyjA10111). No funding bodies had any role in study design, data collection and analysis, decision to publish, or preparation of the manuscript.

\section{Author Contributions}

J.Q. conceived the study. J.Z. and Z.L. performed and checked the available information from eligible articles in this meta-analysis. S.H. analyzed the data. Z.L. prepared Figures 1-8, online suppl. Figures 1-8, and Tables 1-5. J.Z. wrote the main manuscript text. J.Q. reviewed and revised the manuscript. All authors read and approved the final manuscript.

\section{References}

1 Ambati J, Fowler BJ. Mechanisms of age-related macular degeneration. Neuron. 2012 Jul 12;75(1):26-39.

2 Javitt JC, Zhou Z, Maguire MG, Fine SL, Willke RJ. Incidence of exudative age-related macular degeneration among elderly Americans. Ophthalmology. 2003 Aug;110(8):1534-9.

3 Friedman DS, O'Colmain BJ, Muñoz B, Tomany SC, McCarty C, de Jong PT, et al. Prevalence of age-related macular degeneration in the United States. Arch Ophthalmol. 2004 Apr;122(4):564-72.

4 Kawasaki R, Yasuda M, Song SJ, Chen SJ, Jonas JB, Wang JJ, et al. The prevalence of agerelated macular degeneration in Asians: a systematic review and meta-analysis. Ophthalmology. 2010 May;117(5):921-7.

5 Wong CW, Yanagi Y, Lee WK, Ogura Y, Yeo I, Wong TY, et al. Age-related macular degeneration and polypoidal choroidal vasculopathy in Asians. Prog Retin Eye Res. 2016 Jul;53: 107-39.

6 Lopez PF, Sippy BD, Lambert HM, Thach AB, Hinton DR. Transdifferentiated retinal pigment epithelial cells are immunoreactive for vascular endothelial growth factor in surgically excised age-related macular degenera- tion-related choroidal neovascular membranes. Invest Ophthalmol Vis Sci. 1996 Apr; 37(5):855-68.

7 Tong JP, Chan WM, Liu DT, Lai TY, Choy KW, Pang CP, et al. Aqueous humor levels of vascular endothelial growth factor and pigment epithelium-derived factor in polypoidal choroidal vasculopathy and choroidal neovascularization. Am J Ophthalmol. 2006 Mar; 141(3):456-62.

8 Lim LS, Mitchell P, Seddon JM, Holz FG, Wong TY. Age-related macular degeneration. Lancet. 2012 May 5;379(9827):1728-38.

9 Chakravarthy U, Harding SP, Rogers CA, Downes SM, Lotery AJ, Culliford LA, et al. Alternative treatments to inhibit VEGF in age-related choroidal neovascularisation: 2 -year findings of the IVAN randomised controlled trial. Lancet. 2013 Oct 12;382(9900): 1258-67.

10 Bloch SB, Larsen M, Munch IC. Incidence of legal blindness from age-related macular degeneration in denmark: year 2000 to 2010. Am J Ophthalmol. 2012 Feb;153(2):209-13.e2.

11 Kanoff J, Miller J. Pharmacogenetics of the treatment response of age-related macular degeneration with ranibizumab and bevacizum- ab. Semin Ophthalmol. 2013 Sep-Nov;28(56):355-60.

12 Fauser S, Lambrou GN. Genetic predictive biomarkers of anti-VEGF treatment response in patients with neovascular age-related macular degeneration. Surv Ophthalmol. 2015 Mar-Apr;60(2):138-52.

13 Andreoli MT, Morrison MA, Kim BJ, Chen L, Adams SM, Miller JW, et al. Comprehensive analysis of complement factor $\mathrm{H}$ and LOC387715/ARMS2/HTRA1 variants with respect to phenotype in advanced age-related macular degeneration. Am J Ophthalmol. 2009 Dec;148(6):869-74.

14 Kanda A, Chen W, Othman M, Branham KE, Brooks M, Khanna R, et al. A variant of mitochondrial protein LOC387715/ARMS2, not HTRA1, is strongly associated with age-related macular degeneration. Proc Natl Acad Sci U S A. 2007 Oct 9;104(41):16227-32.

15 Kortvely E, Hauck SM, Duetsch G, Gloeckner CJ, Kremmer E, Alge-Priglinger CS, et al. ARMS2 is a constituent of the extracellular matrix providing a link between familial and sporadic age-related macular degenerations. Invest Ophthalmol Vis Sci. 2010 Jan;51(1): 79-88. 
16 Wang G, Scott WK, Whitehead P, Court BL, Kovach JL, Schwartz SG, et al. A novel ARMS2 splice variant is identified in human retina. Exp Eye Res. 2012 Jan;94(1):187-91.

17 Stang A. Critical evaluation of the NewcastleOttawa scale for the assessment of the quality of nonrandomized studies in meta-analyses. Eur J Epidemiol. 2010 Sep;25(9):603-5.

18 Brantley MA Jr, Fang AM, King JM, Tewari A, Kymes SM, Shiels A. Association of complement factor $\mathrm{H}$ and LOC387715 genotypes with response of exudative age-related macular degeneration to intravitreal bevacizumab. Ophthalmology. 2007 Dec;114(12): 2168-73.

19 Orlin A, Hadley D, Chang W, Ho AC, Brown G, Kaiser RS, et al. Association between highrisk disease loci and response to anti-vascular endothelial growth factor treatment for wet age-related macular degeneration. Retina. 2012 Jan;32(1):4-9.

20 Park DH, Kim IT. LOC387715/HTRA1 variants and the response to combined photodynamic therapy with intravitreal bevacizumab for polypoidal choroidal vasculopathy. Retina. 2012 Feb;32(2):299-307.

21 Yamashiro K, Tomita K, Tsujikawa A, Nakata I, Akagi-Kurashige Y, Miyake M, et al. Factors associated with the response of age-related macular degeneration to intravitreal ranibizumab treatment. Am J Ophthalmol. $2012 \mathrm{Jul}$; 154(1):125-36.

22 Abedi F, Wickremasinghe S, Richardson AJ, Islam AF, Guymer RH, Baird PN. Genetic influences on the outcome of anti-vascular endothelial growth factor treatment in neovascular age-related macular degeneration. Ophthalmology. 2013 Aug;120(8):1641-8.

23 Fang K, Tian J, Qing X, Li S, Hou J, Li J, et al. Predictors of visual response to intravitreal bevacizumab for treatment of neovascular age-related macular degeneration. J Ophthalmol. 2013;2013:676049.

24 Hagstrom SA, Ying GS, Pauer GJ, SturgillShort GM, Huang J, Callanan DG, et al. Pharmacogenetics for genes associated with agerelated macular degeneration in the Comparison of AMD Treatments Trials (CATT). Ophthalmology. 2013 Mar;120(3):593-9.

25 Hautamaki A, Kivioja J, Vavuli S, Kakko S, Savolainen ER, Savolainen MJ, et al. Interleukin 8 promoter polymorphism predicts the initial response to bevacizumab treatment for exudative age-related macular degeneration. Retina. 2013 Oct;33(9):1815-27. http:// dx.doi.org/10.1097/IAE.0b013e318285cf92.

26 Kitchens JW, Kassem N, Wood W, Stone TW, Isernhagen R, Wood E, et al. A pharmacogenetics study to predict outcome in patients re- ceiving anti-VEGF therapy in age related macular degeneration. Clin Ophthalmol. 2013;7:1987-93.

27 Lotery AJ, Gibson J, Cree AJ, Downes SM, Harding SP, Rogers CA, et al. Pharmacogenetic associations with vascular endothelial growth factor inhibition in participants with neovascular age-related macular degeneration in the IVAN Study. Ophthalmology. 2013 Dec;120(12):2637-43.

28 Matsumiya W, Honda S, Yanagisawa S, Miki A, Nagai T, Tsukahara Y. Evaluation of clini$\mathrm{cal}$ and genetic indicators for the early response to intravitreal ranibizumab in exudative age-related macular degeneration. Pharmacogenomics. 2014 Apr;15(6):833-43.

29 Park UC, Shin JY, Kim SJ, Shin ES, Lee JE, McCarthy LC, et al. Genetic factors associated with response to intravitreal ranibizumab in Korean patients with neovascular age-related macular degeneration. Retina. 2014 Feb; 34(2):288-97.

30 van Asten F, Rovers MM, Lechanteur YT, Smailhodzic D, Muether PS, Chen J, et al. Predicting non-response to ranibizumab in patients with neovascular age-related macular degeneration. Ophthalmic Epidemiol. 2014 Dec;21(6):347-55.

31 Hata M, Tsujikawa A, Miyake M, Yamashiro $\mathrm{K}$, Ooto S, Oishi A, et al. Two-year visual outcome of ranibizumab in typical neovascular age-related macular degeneration and polypoidal choroidal vasculopathy. Graefes Arch Clin Exp Ophthalmol. 2015 Feb;253(2): 221-7.

32 Kuroda Y, Yamashiro K, Miyake M, Yoshikawa $\mathrm{M}$, Nakanishi H, Oishi A, et al. Factors associated with recurrence of age-related macular degeneration after anti-vascular endothelial growth factor treatment: a retrospective cohort study. Ophthalmology. 2015 Nov; 122(11):2303-10

33 Bardak H, Bardak Y, Ercalik Y, Turkseven Kumral E, Imamoglu S, Gunay M, et al. Effect of ARMS2 gene polymorphism on intravitreal ranibizumab treatment for neovascular age-related macular degeneration. Genet $\mathrm{Mol}$ Res. 2016 Dec 19;15(4).

34 Chaudhary V, Brent M, Lam WC, Devenyi R, Teichman J, Mak M, et al. Genetic risk evaluation in wet age-related macular degeneration treatment response. Ophthalmologica. 2016; 236(2):88-94

35 Kikushima W, Sakurada Y, Yoneyama S, Sugiyama A, Tanabe N, Kume A, et al. Incidence and risk factors of retreatment after threemonthly aflibercept therapy for exudative age-related macular degeneration. Sci Rep. 2017 Mar 7;7:44020
36 Park UC, Shin JY, Chung H, Yu HG. Association of ARMS2 genotype with response to anti-vascular endothelial growth factor treatment in polypoidal choroidal vasculopathy. BMC Ophthalmol. 2017 Dec 7;17(1):241.

37 Yamashiro K, Mori K, Honda S, Kano M, Yanagi $Y$, Obana A, et al. A prospective multicenter study on genome wide associations to ranibizumab treatment outcome for age-related macular degeneration. Sci Rep. 2017 Aug 23;7(1):9196.

38 Mohamad NA, Ramachandran V, Mohd Isa $\mathrm{H}$, Chan YM, Ngah NF, Ching SM, et al. Association of HTRA1 and ARMS2 gene polymorphisms with response to intravitreal ranibizumab among neovascular age-related macular degenerative subjects. Hum Genomics. 2019 Feb 22;13(1):13.

39 Liew G, Mitchell P. Ranibizumab for neovascular age-related macular degeneration. $\mathrm{N}$ Engl J Med. 2007 Feb 15;356(7):747-50.

40 Group CR, Martin DF, Maguire MG, Ying GS, Grunwald JE, Fine SL, et al. Ranibizumab and bevacizumab for neovascular age-related macular degeneration. N Engl J Med. 2011 May 19;364(20):1897-908.

41 Sobrin L, Reynolds R, Yu Y, Fagerness J, Leveziel N, Bernstein PS, et al. ARMS2/ HTRA1 locus can confer differential susceptibility to the advanced subtypes of age-related macular degeneration. Am J Ophthalmol. 2011 Feb;151(2):345-52.e3.

42 Teper SJ, Nowinska A, Pilat J, Palucha A, Wylegala E. Involvement of genetic factors in the response to a variable-dosing ranibizumab treatment regimen for age-related macular degeneration. Mol Vis. 2010 Dec 7;16:2598604 .

43 Fritsche LG, Loenhardt T, Janssen A, Fisher SA, Rivera A, Keilhauer CN, et al. Age-related macular degeneration is associated with an unstable ARMS2 (LOC387715) mRNA. Nat Genet. 2008 Jul;40(7):892-6.

44 Dewan A, Liu M, Hartman S, Zhang SS, Liu DT, Zhao C, et al. HTRA1 promoter polymorphism in wet age-related macular degeneration. Science. 2006 Nov 10;314(5801):989_ 92.

45 Zhou YL, Chen CL, Wang YX, Tong Y, Fang $\mathrm{XL}, \mathrm{Li} \mathrm{L}$, et al. Association between polymorphism rs11200638 in the HTRA1 gene and the response to anti-VEGF treatment of exudative AMD: a meta-analysis. BMC Ophthalmol. 2017 Jun 21;17(1):97.

46 Hu Z, Xie P, Ding Y, Yuan D, Liu Q. Association between variants A69S in ARMS2 gene and response to treatment of exudative AMD: a meta-analysis. Br J Ophthalmol. 2015 May; 99(5):593-8. 